\title{
Akzeptanz von Versorgungsangeboten zur ausschließlichen Fernbehandlung am Beispiel des telemedizinischen Modellprojekts „docdirekt“: ein Mixed-Methods Design
}

\author{
Acceptance of Care Offers for exclusive Remote Treatment \\ Illustrated by the Telemedical Model Project "docdirekt" with a \\ Mixed-Methods Design
}

\section{(ㄷ) (i) $(9)$}

\author{
Autoren \\ Michail von Solodkoff, Christoph Strumann, Jost Steinhäuser
}

Institut

Institut für Allgemeinmedizin, Universitätsklinikum

Schleswig-Holstein, Campus Lübeck

\section{Schlüsselwörter}

Mixed-Methods, e-Health, Telemedizin, Digitalisierung, Versorgungsforschung

Key words mixed-methods, e-health, telemedicine, digitalization, health services research

online publiziert $\quad 2.7 .2020$

\section{Bibliografie}

Gesundheitswesen 2021; 83: 186-194

DOI $10.1055 / a-1173-9903$

ISSN $0941-3790$

(c) 2020. The Author(s).

This is an open access article published by Thieme under the terms of the Creative Commons Attribution-NonDerivative-NonCommercial-License, permitting copying and reproduction so long as the original work is given appropriate credit. Contents may not be used for commercial purposes, or adapted, remixed, transformed or built upon. (https://creativecommons. org/licenses/by-nc-nd/4.0/)

Georg Thieme Verlag KG, Rüdigerstraße 14,

70469 Stuttgart, Germany

Korrespondenzadresse

Michail von Solodkoff

Institut für Allgemeinmedizin

Universitätsklinikum Schleswig-Holstein

Campus Lübeck

Ratzeburger Allee 160

23538 Lübeck

Deutschland

michail.vonsolodkoff@student.uni-luebeck.de

\section{ZUSAMMENFASSUNG}

Ziel der Studie Aktuelle telemedizinische Angebote, wie das in Baden-Württemberg (BW) laufende Modellprojekt „docdirekt“, beinhalten eine Begleitevaluation, die die Implementierung der Anwendung optimieren soll. Anhand der Ergebnisse dieser Evaluation lassen sich Strategien zur Anpassung des Angebots der ausschließlichen Fernbehandlung identifizieren, um dieses langfristig in die Regelversorgung zu integrieren.

Methodik Im ersten Teil der Studie wurden Probanden aus BW in Einzelinterviews zu Erwartungen, fördernden Faktoren und Barrieren bei der Nutzung von Versorgungsangeboten zur ausschließlichen Fernbehandlung befragt. Die mit der qualitativen Inhaltsanalyse nach Mayring explorierten Determinanten wurden dann im zweiten Teil mittels einer quantitativen Befragung von zufällig ausgewählten Bürgern aus BW priorisiert. Ergebnisse 27 Probanden (MW: 51 Jahre, min: 23 Jahre, max: 86 Jahre) nahmen an den Interviews teil. Sie empfanden v. a. die schnelle Erreichbarkeit von medizinischem Fachpersonal als großen Vorteil. Als förderlich wurde u. a. eine „24/7“ Hotline benannt sowie die Möglichkeit elektronische Rezepte oder Arbeitsunfähigkeitsbescheinigungen ausgestellt zu bekommen. Als Barrieren wurden u. a. der Datenschutz und eine fehlende körperliche Untersuchung wahrgenommen. Bei der Fragebogenerhebung wurde von den Teilnehmern ( $n=217$, Rücklauf 10,9\%, MW: 53 Jahre, min: 19 Jahre, max: 88 Jahre) der „schnelle ärztliche Kontakt" bei einer Behandlung per Videotelefonie, gefolgt von der „Möglichkeit einen Spezialisten (Facharzt) vermittelt“ zu bekommen und „einen ersten Therapievorschlag“ zu erhalten als am wichtigsten bewertet. Insgesamt schlossen $n=71$ (34,6\%) die Nutzung eines solchen Angebots für sich aus. Die Nutzungsbereitschaft sinkt mit zunehmendem Alter und abnehmender digitaler Affinität.

Schlussfolgerung Die Studie zeigt, dass Interesse an der Nutzung von Versorgungsangeboten zur ausschließlichen Fernbehandlung besteht. Personen mit Nutzungsabsicht priorisieren dabei die schnelle medizinische Ersteinschätzung sowie die Komfort-Aspekte und betrachten u. a. die Einbindung weiterer Dienstleistungen als förderlich. Es gilt im nächsten Schritt die erhobenen Determinanten in das Angebot einfließen zu lassen. 


\section{ABSTRACT}

Objective Current telemedical services, such as the "docdirekt" project in Baden-Württemberg (BW), are evaluated for optimizing their implementation. Based on the results of this evaluation, strategies for adapting these services for exclusive remote treatment are identified that enable their integration in regular medical care. Methods In the first part of the study, citizens from BW were individually interviewed on their expectations regarding such a project, and the advantages and barriers in the use of services for exclusive remote treatment. Determinants were explored by means of Mayring's qualitative content analysis. In the second part of the study, these determinants were prioritized by using a quantitative survey of randomly selected citizens from BW.

Results A total of 27 subjects (mean age 51 years, min: 23 years, max: 86 years) participated in the interviews. Most importantly, they found the direct accessibility of healthcare professionals a great advantage. A " $24 / 7$ " hotline was found to be beneficial, as well as the possibility to get electronic prescriptions or medical sickness certificates. Privacy and a missing physical examination were perceived as barriers. In the questionnaire, the participants ( $n=217$, return $10.9 \%$, mean age: 53 years, min: 19 years, max: 88 years) rated a "direct medical contact" as the most important, followed by the "possibility to get a specialist" and to receive "a first therapy suggestion". In total, $n=71$ (34.6\%) rejected the future use of such a service. The willingness to use the service decreased with increasing age and decreasing digital skills.

Conclusion The study shows that there is an interest in the use of services for exclusive remote treatment. People who intend to use telemedical service prioritize the fast-initial medical assessment and the comfort aspects and, among other things, consider the inclusion of additional services as beneficial. The next step is to incorporate these determinants into the existing telemedical service.

\section{Einleitung}

Die flächendeckende allgemeinmedizinische Versorgung wird zukünftig vor große Herausforderungen gestellt. Der demografische Wandel bedingt steigende Behandlungszahlen bei teilweise bestehender regionaler Unterversorgung an Hausärzten und einem Altersdurchschnitt von über 60 Jahren bei rund einem Drittel der praktizierenden Ärzte [1-3]. Da bereits jetzt die Mehrheit der Planungsbezirke für Hausärzte offen für eine Niederlassung sind, wird ein dringlicher Handlungsbedarf deutlich [4].

Auch vor diesem Hintergrund entwickelte die Kassenärztliche Vereinigung Baden-Württemberg (KVBW) das telemedizinische Modellprojekt „docdirekt“. Ziel war es die ambulante medizinische Versorgung sowie Notaufnahmen der Krankenhäuser zu unterstützen bzw. zu entlasten [5]. Seit dem Projektbeginn im April 2018 bietet die Onlineplattform gesetzlich Versicherten aus BadenWürttemberg (BW) die Möglichkeit eine medizinische Fernberatung per Telefon, Videotelefonie oder Chat mit niedergelassenen Ärzten aus BW durchzuführen, ohne vorherigen persönlichen Kontakt mit dem Arzt gehabt zu haben. Studien aus Australien und Schweden zeigen, dass Videosprechstunden bei vielen Beratungsanlässen gleichwertig zu einer persönlichen Vorstellung beim Arzt sind $[6,7]$.

Über den Erfolg telemedizinischer Anwendungen entscheiden letztlich die Patienten. Daher gilt es frühzeitig deren Akzeptanz für ein Fernbehandlungsangebot sowie Barrieren und fördernde Faktoren für die Nutzung zu evaluieren [8]. Dadurch können anschließend Strategien zur Anpassung des Angebots identifiziert werden. Telemedizinische Projekte ohne eine solche Begleitevaluation kamen in der Vergangenheit nicht über die Pilotphase hinaus $[9,10]$.

Vor diesem Hintergrund war das Ziel der Studie, Barrieren und fördernde Faktoren für die Nutzung von Angeboten zur ausschließlichen ärztlichen Fernbehandlung zu explorieren und zu priorisieren.

\section{Methoden}

Zur Exploration und Priorisierung der Determinanten wurde ein Mixed-Methods Design gewählt. Im ersten Schritt wurden Determinanten für die Nutzung von Versorgungsangeboten zur ausschließlichen Fernbehandlung aus Patientensicht, mithilfe von qualitativen Einzelinterviews, exploriert. Diese wurden anschließend in einem zweiten Schritt durch quantitative Befragungen priorisiert.

Bei der Evaluation von „docdirekt“ wurde sich dabei am „Model for Assessment of Telemedicine Applications“ (MAST) orientiert, ein im europäischen Raum etabliertes Instrument zur Bewertung telemedizinischer Anwendungen [11].

\section{Einzelinterviews}

In der qualitativen Phase wurden telefonisch Einzelinterviews mit Probanden aus BW von einem Medizinstudenten und Doktoranden des Instituts für Allgemeinmedizin der Universität zu Lübeck (MS) im Zeitraum von November 2018 bis März 2019 durchgeführt. Die Rekrutierung fand durch insgesamt 42 Ärzte, zusammengesetzt aus an „docdirekt“ beteiligten Telemedizinern sowie niedergelassenen Ärzten aus den ursprünglichen Projekt-Modellregionen Stuttgart und dem Landkreis Tuttlingen in BW, statt, die gebeten wurden jeweils 5 zufällig ausgewählte Probanden für ein Telefoninterview zu rekrutieren.

Die Befragung der Probanden, u. a. nach Erwartungen, fördernden Faktoren und Barrieren bei der Nutzung von Versorgungsangeboten zur ausschließlichen Fernbehandlung, wurde mithilfe eines zuvor entwickelten, teilstandardisierten Interviewleitfadens durchgeführt. Ziel war es, die subjektiven Blickwinkel und Standpunkte der Teilnehmer zu erfassen sowie gezielt auf Antworten eingehen zu können [12]. Zudem boten die Einzelinterviews einen geschützten Rahmen und somit die Chance, persönliche Meinungen unbefangen äußern zu können [13].

Es erfolgte eine Transkription der aufgenommenen Gespräche und eine qualitative Auswertung nach Mayring [14]. Zwei Forscher 
(MS, CS: Gesundheitsökonom) codierten unabhängig voneinander das Material. Eine Konsensus-Version der Codes wurde gemeinsam mit einem in der qualitativen Methodik erfahrenen Wissenschaftler (JS: Facharzt für Allgemeinmedizin) erstellt.

\section{Fragebogen}

In der anschließenden quantitativen Untersuchung wurde auf Grundlage der Ergebnisse aus den Einzelinterviews ein Fragebogen erstellt. Dieser beinhaltete 29, aus den vorangegangenen Interviews ausgewählte, explorierte Determinanten, welche die Befragten jeweils mittels einer 6-stufigen Likert-Skala (von „sehr unwichtig“ bis „sehr wichtig“) bewerten konnten. Zusätzlich wurden neben den soziodemografischen Daten der Probanden auch allgemeine Fragen zur ärztlichen Behandlung, Versorgungssituation und generellen Einstellung zur ausschließlichen Fernbehandlung erhoben.

Der postalische Versand der Fragebögen erfolgte an 2000 zufällig ausgewählte Bürger aus der Region Stuttgart und dem Landkreis Tuttlingen, im Zeitraum von Juli bis November 2019. Der Dienstleister für kommunale Informationsverarbeitung in BW (ITEOS) sowie die Stadt Stuttgart stellten hierfür die benötigten Adressen zur Verfügung.

Für die bewerteten Determinanten wurden Mittelwerte berechnet, auf deren Basis für alle Teilnehmer eine Rangordnung der Determinanten erstellt wurde. Zusätzlich wurde, mithilfe von Ordinalen Logistischen Regressionen, der Zusammenhang für jede Determinante, separat mit der Absicht die Videosprechstunde in Zukunft zu nutzen (nein, vielleicht oder ja), geschätzt.

Außerdem wurden zu den einzelnen Determinanten in den separaten Regressionen jeweils Kontrollvariablen berücksichtigt, und zwar zur Soziodemografie (Geschlecht, Alter, Stadt), medizinischen Versorgung (Einschätzung der medizinischen Versorgung, fester Hausarzt ja/nein) und digitalen Affinität, welche mit der Anzahl digitaler Endgeräte approximiert wurde. Zur Vergleichbarkeit der geschätzten Zusammenhänge wurde die Stichprobe auf Teilnehmer begrenzt, die zu jeder Determinante und Kontrollvariable verwertbare Angaben gemacht haben. Die statistischen Analysen im quantitativen Studienteil wurden mit MATLAB, Version 9.4 (R2019b) (The MathWorks, Natick, MA, USA) und mit STATA 15 (StataCorp LLC, College Station, TX, USA) durchgeführt.

\section{Ergebnisse}

\section{Qualitative Ergebnisse}

Insgesamt willigten 34 Probanden in die Teilnahme an einem Interview ein. Sieben Probanden zogen ihr Einverständnis jedoch ohne Angabe von Gründen zurück oder waren aufgrund einer fehlerhaften Kontaktadresse nicht mehr erreichbar, sodass 27 Interviews geführt wurden. Aufgrund der inhaltlichen Sättigung konnte auf eine weitere Rekrutierung von Probanden verzichtet werden. Das durchschnittliche Alter der Teilnehmer betrug 51 Jahre (SD = 18,67; min: 23 J., max: 86 J.), der Anteil der weiblichen Teilnehmer betrug $63 \%$.

Anhand der Interviewleitfragen konnten 47 Determinanten den drei explorierten Hauptkategorien „Erwartungen an ein Fernbehandlungsangebot“, „fördernde Faktoren“ und „Barrieren“ bei der
Nutzung eines Fernbehandlungsangebots zugeordnet werden ( $\triangleright$ Tab. 1).

\section{Erwartungen an ein Fernbehandlungsangebot}

Die Interviewpartner erwarteten von einem Fernbehandlungsangebot eine schnelle Erreichbarkeit von medizinischem Fachpersonal oder Ärzten, die dann Empfehlungen für die ersten therapeutischen Maßnahmen und Entscheidungshilfen für die richtige Weiterbehandlung bzw. Weitervermittlung geben können:

"Ja, und auch die Möglichkeit, [...], schon ohne überhaupt einen Besuch, also ohne Zeitaufwand, schon eine Diagnose zu bekommen. Und auch eventuell einen Therapievorschlag, ohne, dass man viel Zeit investieren muss" (TN 5).

Ein Nutzen liegt laut der Teilnehmer auch im Komfort. Genannt wurden z. B. die örtliche und zeitliche Flexibilität, Wege- und Zeitersparnis, aber auch die Bequemlichkeit bei einer Erkrankung das Haus nicht verlassen zu müssen. Zudem könne durch eine Videosprechstunde auch eine potenzielle Ansteckung in Arztpraxen vermieden werden.

„Ich finde das eine tolle Idee, man ist unabhängig, man kann es von zu Hause aus machen, muss nicht einen Termin ausmachen, dann vor Ort“(TN 8).

\section{Fördernde Faktoren für die Nutzung eines Fernbehandlungsangebots}

Als für die Nutzung förderlich, empfanden die Teilnehmer v. a. eine Erweiterung der Sprechzeiten auf das Wochenende oder eine 24/7-erreichbare Hotline sowie die Möglichkeit elektronische Rezepte (e-Rezept) und Arbeitsunfähigkeitsbescheinigungen (e-AU) ausgestellt zu bekommen.

\section{„Ja, also wenn das mit so Rezepten und Attesten funktionieren würde [...], dann würde ich das aufjeden Fall nutzen“ (TN 6).}

Anforderungen gab es von den Teilnehmern auch an die Ärzte, die die Videosprechstunde durchführen (Teleärzte). Diese sollten speziell für eine telemedizinische Behandlung geschult sein und bei der Anamnese eine standardisierte Vorgehensweise befolgen. Eine Zuweisung immer an denselben Telearzt, sollte es vom Patienten gewünscht werden, könne hierbei ebenso nützlich sein.

\begin{abstract}
„Also von daher wäre es vielleicht auch ganz gut, ja, dieser Arzt wäre vielleicht irgendwie besonders geschult. Um das Gespräch mit mir zu führen. Weil es ist ein fremder Arzt und ich vergesse vielleicht was und er vergisst vielleicht was, was wichtig wäre“" (TN 20).
\end{abstract}

Als vorteilhaft wurde der Zugriff des Telearztes auf eine elektronische Patientenakte (e-Akte) genannt, mit der sich der Telearzt über die Krankenvorgeschichte und eine eventuelle Dauermedikation des Patienten informieren könne. Jedoch solle der Patient selbst darüber entscheiden können, welche Informationen in dieser Akte gespeichert werden. 
- Tab. 1 Ergebnisse der Telefoninterviews.

\begin{tabular}{|c|c|c|}
\hline Kategorien & & Determinanten \\
\hline \multirow[t]{12}{*}{ Erwartungen } & \multirow[t]{4}{*}{ Ersteinschätzung } & Schneller Kontakt zu ärztlichem Personal \\
\hline & & Erste Therapieempfehlung erhalten \\
\hline & & Empfehlung zur Weiterbehandlung \\
\hline & & Beruhigung * \\
\hline & \multirow[t]{5}{*}{ Komfort } & Bequemlichkeit \\
\hline & & Örtliche Flexibilität \\
\hline & & Wegersparnis \\
\hline & & Zeitersparnis \\
\hline & & Zeitliche Flexibilität \\
\hline & \multirow[t]{3}{*}{ Zugang } & Anonymität (unbekannter Arzt) \\
\hline & & Ansteckungsvermeidung \\
\hline & & Niedrige Hemmschwelle \\
\hline \multirow[t]{21}{*}{ Fördernde Faktoren } & \multirow[t]{6}{*}{ Dienstleistung } & E-Rezept \\
\hline & & E-Arbeitsunfähigkeitsbescheinigung \\
\hline & & Infos über Medikamente \\
\hline & & Spezialistentermin \\
\hline & & Abschließend lösbares Problem \\
\hline & & Ärztliche Zweitmeinung \\
\hline & Vertrauen & Vertrauter Arzt \\
\hline & \multirow[t]{2}{*}{ Qualität } & Geschulter Arzt \\
\hline & & Standardisierte Vorgehensweise des Telearztes * \\
\hline & \multirow[t]{3}{*}{ Vernetzung } & Integration von Gesundheits-Apps \\
\hline & & Datenaustausch mit Hausarztpraxis \\
\hline & & Zugriff des Telearztes auf e-Akte \\
\hline & \multirow[t]{4}{*}{ Zugang } & Erweiterte Sprechzeiten \\
\hline & & Kurze Hotline-Nummer \\
\hline & & Eigenes Gerät \\
\hline & & Hilfe bei Bedienung \\
\hline & \multirow[t]{2}{*}{ Datenschutz } & Info über Datenschutzkonzept \\
\hline & & Patientensouveränität über eigene Daten * \\
\hline & Anreize & Bonussystem bei Nutzung * \\
\hline & \multirow[t]{2}{*}{ Bekanntheitsgrad } & Werbemaßnahmen $^{*}$ \\
\hline & & Erfahrungsberichte / Nutzerbewertung einrichten ${ }^{*}$ \\
\hline \multirow[t]{14}{*}{ Barrieren } & \multirow[t]{3}{*}{ Technik } & Datenschutz \\
\hline & & Technische Qualität \\
\hline & & Überforderung mit der Technik im Alter ${ }^{*}$ \\
\hline & \multirow[t]{2}{*}{ Zugang } & Verbindungskosten ${ }^{*}$ \\
\hline & & Neophobie * \\
\hline & \multirow[t]{3}{*}{ Qualität } & Fehlende körperliche Untersuchung * \\
\hline & & Begrenzte Untersuchungsmöglichkeiten ${ }^{*}$ \\
\hline & & Fehlendes Wissen über Krankenvorgeschichte * \\
\hline & Vertrauen & Fremder Arzt * \\
\hline & Bedarf & Ausreichende Versorgung vor Ort * \\
\hline & Informationsvermittlung & Unklares Angebot ${ }^{*}$ \\
\hline & \multirow[t]{3}{*}{ Befürchtungen / Sorgen } & Mehraufwand ${ }^{*}$ \\
\hline & & Arztersatz * \\
\hline & & Fehldiagnosen durch Ferndiagnose ${ }^{*}$ \\
\hline
\end{tabular}


„[...] ich habe das auch nicht so mit verfolgt, aber die Kassen und andere wollen ja auch auf den Patienten Ausweis auch Daten speichern. Und das wäre natürlich gut, diese Daten, so sie dann irgendwann mal gespeichert sind, auch eben dem Telearzt zu übertragen“(TN 16).

Eine pharmakologische Beratung, als Teil des Angebots, wurde ebenfalls als förderlich für die Nutzungsabsicht genannt. Generell sei ein hoher Bekanntheitsgrad eines Angebots wichtig, wie z. B. durch Werbemaßnahmen und Bereitstellung von Erfahrungsberichten.

\section{„Was vielleicht spannend wäre, wäre auch so eine pharmakologi- sche Verbindung. [...] Die Medikamente haben ja nicht nur Wirkung, die haben ja auch irgendwelche Nebenwirkungen und Wechselwirkungen“(TN 26).}

\section{Barrieren bei der Nutzung eines Fernbehandlungsangebots}

Viele Teilnehmer äußerten ihre Besorgnis über die medizinische Qualität eines solchen Angebots. Z. B., dass der Telearzt weder eine körperliche Untersuchung durchführen noch über die Krankenvorgeschichte des Patienten Bescheid wissen könne und insgesamt nur eingeschränkte Kommunikationsmöglichkeiten zur Verfügung stünden.

„Ja, das denke ich schon, weil letztendlich bleibt ein bisschen die körperliche Untersuchung wahrscheinlich auf der Strecke. Die Diagnostik ist vielleicht für den Arzt auch schwierig, der mir da am Bildschirm gegenübersitzt“(TN 20).

Teilnehmer nannten auch, dass, aufgrund einer bestehenden guten medizinischen Versorgung vor Ort, eine Konsultation eines Telearztes überhaupt nicht notwendig sei und v. a. ältere Patienten mit der Technik überfordert sein könnten bzw. sind.

„Also ich denke, in einem gewissen Alter, so wie ich jetzt bin, dass man [überlegendes Geräusch] das vielleicht auch gar nicht mehr so versteht am Telefon, wie wenn ich jetzt persönlich mit dem Arzt sprechen kann" (TN 18).

Neben einer allgemeinen Datenschutzproblematik wurde auch eine Unwissenheit beklagt, in welchen medizinischen Fällen dieses Angebot überhaupt genutzt werden könne.

„Gut die Frage ist für mich dann so ein bisschen, die sich stellt, vielleicht ganz grundsätzlich, wann rufe ich bei dieser Hotline an. Also wenn ich jetzt eine Erkältung habe und ein Attest brauche oder so. Oder wenn ich tatsächlich, [überlegendes Geräusch] weiß ich nicht, so ein bisschen mehr in Richtung Notfall, oder [überlegendes Geräusch] ja, also weiß ich nicht“ (TN 12).

Es bestand die Befürchtung, dass eine vorherige Konsultation eines Telearztes, aufgrund einer medizinischen Problematik, letztendlich Mehraufwand bedeuten würde, da man meistens dennoch in einer Arztpraxis vorstellig werden müsse.
„Also dann, ja, bringt es zwar insofern etwas, dass man dann das weiß, dass man dann aufjeden Fall jetzt doch zum Arzt muss, aber es erleichtert es halt insofern dann ja doch nicht ganz, weil man den Weg ja dann trotzdem wiederhat" (TN 3).

Aus der qualitativen Analyse konnten insgesamt 29 Determinanten ausgewählt und exploriert werden, deren Priorisierung im Folgenden beschrieben wird.

\section{Deskriptive Ergebnisse}

Von den 2000 postalisch verschickten Fragebögen wurden 217 zurückgesandt (Rücklaufquote: 10,9\%). Alle Rückläufer waren gültig und konnten in die Analyse eingeschlossen werden. Das durchschnittliche Probandenalter betrug 53 Jahre (SD = 17,2; min: 19 J., max: 88 J.). T Tab. 2 zeigt u. a. die soziodemografischen Merkmale der Studienteilnehmer, die Nutzungsabsicht der ärztlichen Behandlung per Videotelefonie, Einschätzungen zur medizinischen Vesorgung und zu ihrer digitalen Affinität.

Die Teilnehmer wurden nach ihrer zukünftigen allgemeinen Nutzungsabsicht der ärztlichen Behandlung per Videotelefonie befragt ( Tab. 2). Insgesamt schlossen 34,6\% die Nutzung für sich aus. Am geringsten war das Interesse bei der Altersgruppe > 65 Jahren, in der $60 \%$ der Teilnehmer angaben, Videotelefonie nicht nutzen zu wollen. In dieser Altersgruppe haben 71,7\% einen Internetzugang. Umgekehrt beabsichtigte die Mehrheit (59\%) innerhalb der Altersgruppe < 35 Jahren, das Angebot zu nutzen. Für die weiteren Analysen wurden 37 Teilnehmer ausgeschlossen, da sie nicht zu jeder Determinante und Kontrollvariable verwertbare Angaben machten.

\section{Bewertung der Determinanten}

Von den in den Interviews ausgewählten, explorierten 29 Determinanten zu den Themenbereichen „Erwartungen“, „fördernde Faktoren“ und „Barrieren“ bei der Nutzung eines Fernbehandlungsangebots, welche die Befragten jeweils mittels einer 6-stufigen Likert Skala (von 1 „sehr unwichtig“ bis 6 „sehr wichtig“) bewerten konnten, sind die Mittelwerte berechnet worden. Auf dieser Basis wurde für alle Teilnehmer eine Rangordnung der Determinanten erstellt ( $\triangleright$ Tab. 3).

Am persönlich wichtigsten bei einer ärztlichen Behandlung per Videotelefonie wurde von den Teilnehmern der „schnelle ärztliche Kontakt“ (MW 5,37 von 6) empfunden. Gefolgt von der Möglichkeit einen „Spezialistentermin“ vermittelt zu bekommen (MW 5,1) und „eine erste Therapieempfehlung“ (MW 5,02) zu erhalten. Auch der „Datenschutz“ (MW 4,93) und „geschulte Teleärzte“ (MW 4,88) wurden als wichtig empfunden. Einen mittleren Rang belegten die Aspekte „Erreichbarkeit am Wochenende“ (MW 4,15) und „die Ausstellung von elektronischen Rezepten“ (MW 4,08). Die Aspekte „Ausstellung von elektronischen Arbeitsunfähigkeitsbescheinigungen“ (MW 3,27) und „die Bequemlichkeit, z. B. nicht das Haus verlassen und im Wartezimmer sitzen zu müssen“ (MW 3,69) wurden insgesamt als weniger wichtig bewertet.

\section{Zusammenhang der Determinanten mit der Nutzungsabsicht}

Die Ergebnisse der Ordinalen Logistischen Regression sind ebenfalls in > Tab. 3 abgebildet. Je älter die Probanden waren und je geringer die digitale Affinität (gemessen an der Anzahl der Endgerä- 
- Tab. 2 Deskriptive Statistiken.

\begin{tabular}{|c|c|c|c|c|}
\hline & alle & $<35 \mathrm{~J}$. & $35-65 \mathrm{~J}$. & $>65 \mathrm{~J}$. \\
\hline \multicolumn{5}{|l|}{ Stichprobenumfang } \\
\hline Anzahl Beobachtungen & $217^{* *}$ & 39 & 113 & 60 \\
\hline Anteil Beobachtungen (\%) & 100,0 & 18,0 & 52,1 & 27,7 \\
\hline Anteil der ausgeschlossenen Teilnehmer (\%) ${ }^{*}$ & 17,1 & 7,7 & 9,7 & 30,0 \\
\hline \multicolumn{5}{|l|}{ Soziodemografie } \\
\hline Alter & 53,4 & 28,4 & 51,1 & 73,8 \\
\hline weiblich (Anteil in \%) & 54,4 & 48,7 & 55,8 & 55,0 \\
\hline Region Stuttgart (Anteil in \%) & 53,5 & 69,2 & 50,0 & 51,7 \\
\hline Stadt (Anteil in \%) & 67,8 & 71,8 & 60,7 & 78,3 \\
\hline chronische Erkrankung (Anteil in \%) & 28,4 & 5,1 & 23,2 & 53,5 \\
\hline \multicolumn{5}{|l|}{ Nutzung der ärztlichen Behandlung per Videotelefonie (Anteile in \%) } \\
\hline \multicolumn{5}{|l|}{ Ich beabsichtige in Zukunft Videotelefonie zu nutzen } \\
\hline Nein & 34,6 & 12,8 & 29,2 & 60,0 \\
\hline Vielleicht & 30,9 & 28,2 & 39,8 & 18,3 \\
\hline Ja & 32,7 & 59,0 & 31,0 & 16,7 \\
\hline Ich habe bereits Videotelefonie genutzt & 1,4 & 0,0 & 1,8 & 1,8 \\
\hline \multicolumn{5}{|l|}{ Medizinische Versorgung } \\
\hline \multicolumn{5}{|l|}{ Wie lange sind Sie bereits Patient/in bei Ihrem Hausarzt? (Anteile in \%) } \\
\hline Ich habe keinen festen Hausarzt & 8,5 & 15,4 & 9,7 & 1,8 \\
\hline weniger als 5 Jahre & 19,8 & 35,9 & 20,4 & 8,3 \\
\hline 5 Jahre oder länger & 69,6 & 48,7 & 69,9 & 83,3 \\
\hline Wichtigkeit einen festen Hausarzt zu haben (1-6) & 5,4 & 4,6 & 5,4 & 5,9 \\
\hline Wichtigkeit des unmittelbaren persönlichen Kontakts zum Hausarzt vor Ort (1-6) & 5,2 & 4,6 & 5,2 & 5,7 \\
\hline Wichtigkeit, dass der Arzt auch auf die Körpersprache eingehen kann (1-6) & 4,9 & 4,3 & 5,0 & 5,2 \\
\hline Bewertung der medizinischen Versorgung im Allgemeinen (1-6) & 4,7 & 4,7 & 4,6 & 5,1 \\
\hline Bewertung der Wartezeit beim Hausarzt (1-6) & 4,8 & 4,8 & 4,7 & 4,8 \\
\hline Bewertung der Wartezeit beim Spezialisten (1-6) & 3,2 & 3,0 & 2,9 & 3,7 \\
\hline Wartezeit im Wartezimmer beim Hausarzt (ohne Termin) (min) & 46,6 & 45,4 & 47,0 & 46,5 \\
\hline Wegezeit zum Hausarzt (min) & 13,7 & 15,4 & 13,2 & 13,7 \\
\hline \multicolumn{5}{|l|}{ Digitale Affinität } \\
\hline Internetzugang (Anteil in \%) & 90,7 & 100,0 & 97,4 & 71,7 \\
\hline Anzahl digitaler Endgeräte & 2,4 & 2,8 & 2,7 & 1,7 \\
\hline Anzahl der Internetrecherchen innerhalb eines Jahres zu Gesundheitsfragen & 17,6 & 15,0 & 17,5 & 20,6 \\
\hline
\end{tabular}

te), desto geringer war auch die Nutzungsabsicht. Diese nahm auch ab, je besser die medizinische Versorgung vor Ort beurteilt wurde. Personen, die ihren Wohnort als städtisch charakterisierten, zeigten eine höhere Nutzungsabsicht von Fernbehandlungsangeboten. Jedoch war dieser Effekt nur auf dem $10 \%$ Niveau signifikant.

Der stärkste Zusammenhang zeigte sich zwischen Nutzungsabsicht und dem Zugriff auf eine elektronische Patientenakte durch den behandelnden Telearzt. Je höher die Teilnehmer diesen Aspekt bewertet haben, desto höher war deren Nutzungsabsicht. Weitere Determinanten, die von Probanden mit einer Nutzungsabsicht von Videosprechstunden als wichtiger bewertet wurden, sind überwiegend Komfort-Aspekte, wie die „Zeit- und Wegeersparnis“, die „Bequemlichkeit“, die „örtliche und zeitliche Flexibilität“, aber auch die Integration von Dienstleistungen, wie die Ausstellung von „e-
Rezepten“, „elektronischen Arbeitsunfähigkeitsbescheinigungen“ und „Informationen über Medikamente“.

\section{Diskussion}

Anhand der Auswertungen der qualitativen Interviews und anknüpfenden Fragebogenerhebung wurde ein Meinungsbild über die ärztliche Behandlung per Videotelefonie in BW sowie die Relevanz einiger Aspekte, die ein Fernbehandlungsangebot bieten sollte, untersucht.

Die Ergebnisse passen weitgehend zu den Ergebnissen der Versichertenbefragung der KBV von 2019 und zeigen, dass mit 34,6\% der Anteil derjenigen, die ein solches Angebot nicht nutzen wollen, eher gering ist [15]. Das bestätigt wiederum die Vermutung von Ärzten, die, laut einer Umfrage im Auftrag der Bundesärztekam- 
- Tab. 3 Regressionsanalyse: Nutzungsabsicht Telemedizin.

\begin{tabular}{|c|c|c|c|c|c|c|}
\hline Rang & Determinanten & MW & Ordinales Logit ${ }^{b}$ & \multicolumn{2}{|c|}{ Kontroll- variablen (KV) } & Ordinales Logit $^{\mathrm{a}}$ \\
\hline & & & (KV + Det.) & & & (nur KV) \\
\hline 1 & schneller Kontakt & 5,37 & $0,46^{* *}$ & & & \\
\hline 2 & Spezialistentermin & 5,10 & $0,34^{* *}$ & \multicolumn{2}{|l|}{ weiblich } & $-0,46$ \\
\hline 3 & Therapieempfehlung & 5,02 & $0,43 * * *$ & \multicolumn{2}{|l|}{ Alter } & $-0,04 * * *$ \\
\hline 4 & ärztliche Empfehlung zur Weiterbehandlung & 4,95 & $0,34^{* *}$ & \multicolumn{2}{|c|}{ medizinische Versorgung } & $-0,28^{* *}$ \\
\hline 5 & Datenschutz & 4,93 & 0,01 & \multicolumn{2}{|c|}{ Stadt } & $0,57^{*}$ \\
\hline 6 & geschulter Arzt & 4,88 & 0,11 & \multicolumn{2}{|c|}{ kein Hausarzt } & 0,73 \\
\hline 7 & Zeitersparnis & 4,55 & $0,51 * * *$ & \multirow{2}{*}{\multicolumn{2}{|c|}{ Anzahl digitaler Endgeräte }} & \multirow[t]{2}{*}{$0,61^{* * *}$} \\
\hline 8 & niedrige Hemmschwelle & 4,50 & 0,17 & & & \\
\hline 9 & eigenes Gerät & 4,49 & $0,48^{* * *}$ & \multirow{4}{*}{$\begin{array}{l}\text { Schwellen- } \\
\text { werte }\end{array}$} & \multirow{4}{*}{$\begin{array}{l}\alpha_{1} \\
\alpha_{2}\end{array}$} & \multirow{4}{*}{$\begin{array}{l}-2,29 * * \\
-0,42\end{array}$} \\
\hline 10 & technische Qualität & 4,48 & $0,58^{* * *}$ & & & \\
\hline 11 & zeitliche Flexibilität & 4,44 & $0,52 * * *$ & & & \\
\hline 12 & ärztliche Zweitmeinung & 4,39 & $0,24^{* *}$ & & & \\
\hline 13 & Zugriff des Telearztes auf E-Akte & 4,37 & $0,68^{* * *}$ & \multirow{3}{*}{\multicolumn{2}{|c|}{ Beobachtungen }} & \multirow{3}{*}{180} \\
\hline 14 & örtliche Flexibilität & 4,28 & $0,47^{* * *}$ & & & \\
\hline 15 & Vernetzung mit Hausarztpraxis & 4,27 & $0,44^{* * *}$ & & & \\
\hline 16 & vertrauter Arzt & 4,26 & 0,14 & & & \\
\hline 17 & Erreichbarkeit am Wochenende & 4,15 & $0,34 * * *$ & & & \\
\hline 18 & elektronisches Rezept & 4,08 & $0,55^{* * *}$ & & & \\
\hline 19 & abschließend lösbares Problem & 4,08 & $0,30 * * *$ & & & \\
\hline 20 & Info über Datenschutzkonzept & 4,06 & $-0,03$ & & & \\
\hline 21 & Ansteckungsvermeidung & 4,01 & 0,04 & & & \\
\hline 22 & Hilfe bei der Bedienung & 3,98 & 0,14 & & & \\
\hline 23 & Infos über Medikamente & 3,86 & $0,50 * * *$ & & & \\
\hline 24 & Wegersparnis & 3,84 & $0,49 * * *$ & & & \\
\hline 25 & kurze Hotline-Nummer & 3,81 & $0,30 * * *$ & & & \\
\hline 26 & Bequemlichkeit & 3,69 & $0,44^{* * *}$ & & & \\
\hline 27 & $\begin{array}{l}\text { elektronische Arbeitsunfähigkeitsbescheini- } \\
\text { gung }\end{array}$ & 3,27 & $0,18^{*}$ & & & \\
\hline 28 & Integration von Apps & 3,02 & $0,26^{* * *}$ & & & \\
\hline 29 & Anonymität & 2,97 & 0,07 & & & \\
\hline
\end{tabular}

mer (BÄK), die Bedeutung der Telemedizin im Gesundheitswesen als zunehmend beurteilten [16]. Unter den besonderen Umständen der Corona-Pandemie erlebt die Bereitschaft, Videotelefonie zu verwenden, zudem aktuell eine erhebliche Steigerung [17]. Als Zugehörige der „digital natives“ wollen besonders die Teilnehmer unter 35 Jahren mit 87,2 \% in Zukunft telemedizinische Leistungen in Anspruch nehmen oder können es sich zumindest vorstellen. Die Nutzung technischer Geräte und des Internet ist in dieser Altersgruppe eine Selbstverständlichkeit, weswegen die vermehrte Akzeptanz der Videotelefonie bestehen könnte [16]. Die Kluft der digitalen Affinität im Vergleich zu den über 65-jährigen ist in unserer Befragung auch vorhanden, es gibt jedoch Hinweise, dass diese insgesamt schwindet [18].

Von den Teilnehmern der Umfrage haben 1,4\% eine Videosprechstunde genutzt und einigen Interviewteilnehmern war nicht genau bekannt, für welche Situationen die Nutzung der Fernbe- handlung überhaupt gedacht sei. Bei dem Projekt „docdirekt“ wurde zu Beginn bewusst auf Werbekampagnen verzichtet [19]. Laut Studien, die das mit „docdirekt“ vergleichbare englische Fernbehandlungsangebot „NHS Direct“ evaluierten, ist aber, gerade für die Akzeptanzsteigerung, ein flächendeckender Bekanntheitsgrad des Angebots und eine Kenntnisvermittlung der konkreten Nutzungsmöglichkeiten von großer Bedeutung [20].

Die Aspekte „schneller Kontakt mit einem Arzt“ und „Therapieempfehlung“ wurden von den Teilnehmern als am wichtigsten eingestuft. Eine Studie zum Nutzungsverhalten von Notaufnahmen von 2017 zeigte, dass mehr als die Hälfte der Patienten ihre Behandlung als nicht dringend einschätzen [21]. Die Möglichkeit, dass Patienten im Vorhinein ihre Beschwerden telemedizinisch abklären und eine fachliche Einschätzung über die Behandlungsdringlichkeit bzw. -notwendigkeit von einem Telearzt erhalten können, könnte daher Ressourcen in Notfallambulanzen und möglicherweise auch 
Kosten einsparen. Einige Interviewteilnehmer äußerten, dass alleine der Kontakt mit medizinischem Personal bei vielen Beschwerden häufig für ausreichende Beruhigung und damit Linderung der Beschwerden sorge. Das bestätigen die Ergebnisse aus einer Studie, die zeigen, dass mit der ausschließlichen Fernbehandlung ca. $50 \%$ der Anrufe abschließend geklärt werden können und dies zur Entlastung medizinischer Einrichtungen beitragen kann [22].

Bisher ist „docdirekt“ von Montag bis Freitag zwischen 9-19 Uhr erreichbar, wird jedoch in über $25 \%$ der Fälle am Wochenende versucht zu erreichen [23]. Dies spiegelt sich auch in den Ergebnissen der Interviews wider, in denen eine Erweiterung der Erreichbarkeit bzw. eine 24/7-Hotline als förderlich für die Nutzung beschrieben wurde.

Seit November 2019 besteht die Möglichkeit im Rahmen von „docdirekt“ e-Rezepte auszustellen. Ein Faktor, der als förderliche Determinante identifiziert wurde. Dies passt auch zu der politischen Einschätzung, dass der Erfolg telemedizinischer Projekte abhängig von der Integration eines e-Rezeptes ist [24].

Der Datenschutz wurde von den Probanden als wichtig eingestuft. Ein Zusammenhang mit der Nutzungsabsicht von Videosprechstunden konnte jedoch nicht gefunden werden. Dies ist daher bemerkenswert, da in Deutschland die Sorge über Sicherheit, sowohl bei der Datenverarbeitung als auch -übermittlung, gesellschaftlich sonst einen großen Stellenwert hat [25]. Ein Dienstleister muss selbstverständlich hohe Anforderungen an den Schutz der persönlichen und medizinischen Daten stellen und das Konzept mit den Anwendern kommunizieren. Länder wie Estland, das zu den Spitzenreitern der Digitalisierung zählt, zeigen schon seit Jahren, dass sich der medizinische Datenaustausch zwischen allen Akteuren im Gesundheitswesen auch mit einem sicheren Datenschutz vereinbaren lässt [26].

Die Teilnehmer bewerteten, unabhängig von deren Nutzungsabsicht, auch die Schulung des telemedizinischen Personals als wichtig. Dies könnte für eine mögliche Barriere bei Probanden ohne Nutzungsabsicht sprechen, die fehlende Kenntnisse telemedizinischer Fertigkeiten bzw. mangelnde Erfahrung des Personals mit telemedizinischen Behandlungen befürchten. Das bestätigt das Vorhaben der BÄK, die Qualität einer Fernbehandlung zukünftig sicherstellen zu wollen. Beispielsweise mit einer Heranführung der Ärzte an telemedizinische Methoden mittels Fortbildungen oder durch die Aufnahme telemedizinischer Fertigkeiten in das Curriculum des Medizinstudiums [27, 28].

\section{Stärken und Schwächen}

Diese ist die erste wissenschaftliche Arbeit über das Thema „Akzeptanz von ausschließlichen Fernbehandlungsangeboten “ in Deutschland. Ein Selektionsbias der Studienprobanden, insbesondere bei der quantitativen Befragung, kann aufgrund des geringen Rücklaufs nicht ausgeschlossen werden. Die Ergebnisse müssen daher vorsichtig interpretiert werden und sind nicht verallgemeinerbar.

\section{Schlussfolgerung}

Die Studie zeigt wesentliche Determinanten für die Implementierung von Angeboten zur ausschließlichen Fernbehandlung, wie „docdirekt“, auf. Akzeptanz und Nutzungsabsicht sind innerhalb der Bevölkerung der Modellregionen Stuttgart und Tuttlingen, die stellvertretend für städtische bzw. ländliche Regionen von BW stehen, überwiegend vorhanden. Da v. a. in ländlichen Regionen neue Versorgungskonzepte eine zunehmende Rolle bei der hausärztlichen Versorgung spielen könnten, sollte das Ergebnis unserer Studie, dass hier die Nutzungsabsicht tendenziell geringer als im städtischen Raum ist, in Folgestudien besonders bedacht werden. Im nächsten Schritt innerhalb der Begleitevaluation von „docdirekt“ werden aus den Ergebnissen konkrete Handlungsempfehlungen erarbeitet und diese in das zukünftige Fernbehandlungsangebot übernommen.

\section{Danksagung}

Die Autoren danken allen an der Erhebung Beteiligten für Ihre Unterstützung, insbesondere der Kassenärztlichen Vereinigung Baden-Württemberg, den Städten Stuttgart und Tuttlingen sowie dem Landratsamt Tuttlingen.

\section{Interessenkonflikt}

Die Autoren geben keinen Interessenkonflikt an. Die Studie wurde finanziell durch die Kassenärztliche Vereinigung Baden-Württemberg und das Ministerium für Soziales und Integration Baden-Württemberg unterstützt. Die Studie erfolgte weisungsunabhängig.

Literatur

[1] Kassenärztliche Bundesvereinigung (KBV). Deutschlandweite Projektion 2030 - Arztzahlentwicklung in Deutschland. Im Internet: https://www.kbv.de/media/sp/2016_10_05_Projektion_2030_Arztzahlentwicklung.pdf; Stand: 23.04.2020

[2] Klose JRI. Ärzteatlas 2017. Daten zur Versorgungsdichte von Vertragsärzten. Wissenschaftliches Institut der AOK (WidO); 2017

[3] Kassenärztliche Bundesvereinigung (KBV). Statistische Informationen aus dem Bundesarztregister, Bundesgebiet insgesamt (Stand: 31.12.2019). Im Internet: https://www.kbv.de/media/sp/2019_12_31_ BAR_Statistik.pdf; Stand: 23.04.2020

[4] Kassenärztliche Vereinigung Baden-Württemberg. Offen oder gesperrt? Niederlassungsmöglichkeiten nach Fachgruppen (Stand: 12.02.2020). Im Internet: https://www.kvbawue.de/praxis/niederlassung/bedarfsplanung-offen-oder-gesperrt/?tx_kvbwversorgungsstandv2_ext1\%5Btyp \%5D = Hausärzte\&tx_kvbwversorgungsstandv2_ ext $1 \% 5$ Bcontroller \%5D = Versorgungsstand\&cHash = 6ffa57d150dbdef e8cefbf14cbfccbc8\#doctorgroups; Stand: 26.04.2020

[5] digital@bw. Projekte - docdirekt. Im Internet: https://www.digital-bw. de/-/docdirekt; Stand: 25.11.2019

[6] Jiwa M, Meng X. Video consultation use by Australian general practitioners: video vignette study. J Med Internet Res 2013; 15: e117. doi:10.2196/jmir.2638

[7] Johansson AM, Lindberg I, Soderberg S. Patients' Experiences with Specialist Care via Video Consultation in Primary Healthcare in Rural Areas. Int J Telemed Appl 2014; 2014: 143824. doi:10.1155/ 2014/143824

[8] Schultz C, Gemünden HG, Salomo S. Akzeptanz der Telemedizin. Darmstadt: Minerva; 2005 
[9] Arnold K, Scheibe M, Müller O et al. Grundsätze für die Evaluation telemedizinischer Anwendungen - Ergebnisse eines systematischen Reviews und Konsens-Verfahrens. Zeitschrift Für Evidenz, Fortbildung und Qualität Im Gesundheitswesen 2016; 117: 9-19

[10] Allner R, Wilfling D, Kidholm K et al. Telemedizinprojekte im ländlichen Raum Deutschlands. Eine systematische Bewertung mit dem „Modell zur Evaluation von telemedizinischen Anwendungen“. Zeitschrift für Evidenz, Fortbildung und Qualität im Gesundheitswesen. 2019; 141-142: 89-95

[11] Kidholm K, Ekeland AG, Jensen LK et al. A model for assessment of telemedicine applications: mast. Int ] Technol Assess Health Care 2012; 28: 44-51. doi:10.1017/S0266462311000638

[12] Flick U, von Kardoff E, Keupp H et al. Handbuch Qualitative Sozialforschung: Grundlagen, Konzepte, Methoden und Anwendungen Beltz Psychologie Verlags Union; 2012

[13] Döring N, Bortz J. Forschungsmethoden und Evaluation in den Sozial- und Humanwissenschaften. Berlin; 2014

[14] Mayring P. Qualitative Inhaltsanalyse. In: May G, Mruck K, Hrsg. Handbuch Qualitative Forschung in der Psychologie. Wiesbaden: Springer Fachmedien Wiesbaden GmbH; 2010: 601-613

[15] Kassenärztliche Bundesvereinigung (KBV). Versichertenbefragung der Kassenärztlichen Bundesvereinigung 2019, Ergebnisse einer repräsentativen Bevölkerungsumfrage. Im Internet: https://www.kbv. de/media/sp/Berichtband_Ergebnisse_der_Versichertenbefragung_2019.pdf; Stand: 23.01.2020

[16] Institut für Demoskopie Allensbach. Der Einsatz von Telematik und Telemedizin im Gesundheitswesen. Im Internet: https://www. bundesaerztekammer.de/fileadmin/user_upload/downloads/ pdf-Ordner/Telemedizin_Telematik/Telemedizin/eHealth_Bericht_ kurz_final_1_.pdf; Stand: 23.01.2020

[17] Krüger-Brand HE. Telemedizin: Sichere Alternative per Video. Deutsches Ärzteblatt 2020; 117: 678-679

[18] Haluza D, Naszay M, Stockinger A et al. Digital Natives Versus Digital Immigrants: Influence of Online Health Information Seeking on the Doctor-Patient Relationship. Health Commun 2017; 32: 1342-1349. doi:10.1080/10410236.2016.1220044
[19] Ärzte Zeitung. Telemedizin-Modellprojekt DocDirekt - Oft wird gechattet mit dem Arzt (18.07.2018). Im Internet: https://www. aerztezeitung.de/Wirtschaft/Oft-wird-gechattet-mit-demArzt-230263.html; Stand: 10.11.2019

[20] Cook E], Randhawa G, Large $S$ et al. Barriers and facilitators to using NHS Direct: a qualitative study of 'users' and 'non-users'. BMC Health Serv Res 2014; 14: 487. doi:10.1186/s12913-014-0487-3

[21] Scherer M, Luhmann D, Kazek A et al. Patients Attending Emergency Departments. Dtsch Arztebl Int 2017; 114: 645-652. doi:10.3238/ arztebl.2017.0645

[22] Stacey D, Noorani H, Fisher A et al. A clinical and economic review of telephone triage services and survey of Canadian call centre programs. Ottawa: Canadian Coordinating Office for Health Technology Assessment; 2004

[23] Becker E. DocDirekt steht vor nächster Ausbaustufe (17.10.2018).Im Internet: https://www.aerztezeitung.de/Politik/DocDirekt-steht-vornaechster-Ausbaustufe-225422.html; Stand: 10.11.2019

[24] Winnat C. Der Gesundheitsminister will das elektronische Rezept (21.05.2018). Im Internet: https://www.aerztezeitung.de/Wirtschaft/ Der-Gesundheitsminister-will-das-elektronische-Rezept-312841.html; Stand 10.11.2019

[25] Garstka H. Datenschutz bei Telemedizin. Der Hautarzt 2019; doi: https://doi.org/10.1007/s00105-019-4380-1

[26] Thiel R, Deimel L, Schmidtmann D et al. SmartHealthSystems: Auszug Estland. In: SmartHealthSystems: Bertelsmann Stiftung 2018

[27] Pathipati AS, Azad TD, Jethwani K. Telemedical Education: Training Digital Natives in Telemedicine. J Med Internet Res 2016; 18: e193. doi:10.2196/jmir.5534

[28] Krüger-Brand HE. Weg frei für die Telemedizin. Deutsches Ärzteblatt 2018; 20-21: 965-968 